\title{
THE COMPUTATIONAL EFFICIENCY OF WALSH APPROXIMATION FOR TWO-DIMENSIONAL VOLTERRA INTEGRAL EQUATIONS
}

\author{
S. Anderyance \\ Department of Mathematics, Faculty of Science, \\ K. N. Toosi University of Technology, \\ Tehran, Iran \\ anderaince@kntu.ac.ir \\ M. Hadizadeh \\ Department of Mathematics, Faculty of Science, \\ K. N. Toosi University of Technology, \\ Tehran, Iran \\ hadizadeh@kntu.ac.ir \\ Communicated by A. Laptev \\ Received March 11, 2009 \\ Revised May 6, 2010
}

\begin{abstract}
In this research, we give details of a new numerical method for the approximate solution of a general two-dimensional Volterra integral equation, using the discontinuous wavelet packets e.g. Walsh functions. The double Walsh approximation we have adopted utilizes a simple robust numerical scheme for approximate solution of the equations. The twodimensional operational matrix of integration for each subinterval $\left[\frac{i-1}{m}, \frac{i}{m}\right]$ is explicitly constructed, where $m$ is a power of 2 . Finally the reliability and efficiency of the proposed scheme are demonstrated by some numerical results.
\end{abstract}

Keywords: Walsh function; operational matrix; two-dimensional Walsh transformation; integral equations; numerical treatments.

AMS Subject Classification: 65R20, 34A25

\section{Introduction}

In recent years, the Walsh theory has been innovated and investigated to various fields of applied sciences e.g. signal processing, communication and pattern recognition [18], transform spectroscopy [10], heart rate, optimal linear systems [17], variational problems $[6,20]$, etc. The basic properties and notations of Walsh functions may be found in $[18,21,9]$ and references therein. This paper is concerned with the development of efficient numerical method based on double Wlash transformation 
for the solution of two-dimensional Volterra integral equations of the second kind

$$
u(x, y)=f(x, y)+\int_{0}^{x} \int_{0}^{y} k(x, y, s, t) u(s, t) d s d t, \quad(x, y) \in D,
$$

where the known functions $f(x, y)$ and $k(x, y, s, t)$ are defined, respectively, on $D:=[0,1] \times[0,1]$ and $S:=\{(x, y, s, t): 0 \leq s \leq y \leq 1, \quad 0 \leq t \leq x \leq 1\}$ and $u(x, y)$ is a solution to be determined. This equation may arise from certain hyperbolic differential equations (see [8], for an equivalent formulation of the Darboux problem). Actually, a few approximate methods for (1.1) are known. Beltyukov et al. in [1] proposed a class of explicit Runge-Kutta type methods of order 3. Bivariate cubic spline functions method was obtained by Singh in [23]. In [4] an exhaustive analysis of polynomial spline collocation and iterated methods was given by Brunner and Kauthen. The asymptotic error expansion of collocation and iterated collocation as well as Galerkin and iterated Galerkin solutions for two-dimensional linear and nonlinear Volterra integral equations were obtained by Guoqiang et al. in [14, 13]. Here, we restrict our attention to the approximate solution of the linear two-dimensional Volterra integral equations by the Walsh function spectral approach. There has been considerable interest in solving integral equations using techniques which involve Walsh functions. A summary of the historical developments of the Walsh approximation for integral equations may be found in $[15,3]$ where an excellent bibliography on Walsh functions and its applications is also given in [9]. One of the motivations for these developments is that these methods usually involve the use of fast Walsh Fourier transform, which is faster than the corresponding transforms such as the trigonometric fast Fourier transform. Also, Walsh functions appear to be easily incorporated into a wide variety of robust general purpose algorithms.

\section{Preliminaries and Basic Idea}

Let $f \in L^{2}[0,1)$, then $f(x)$ can be expanded as a series of Walsh functions $f(x)=$ $\sum_{i=0}^{\infty} c_{i} W_{i}(x)$, where $c_{i}=\int_{0}^{1} f(x) W_{i}(x) d x$. It is well known that its integral from 0 to $x$ have Walsh series with coefficients of $b_{i}$, where $\int_{0}^{x} f(t) d t=\sum_{i=0}^{\infty} b_{i} W_{i}(x)$. If we truncate to $m=2^{n}$ terms and use to obvious vector notation, then integration is performed by matrix multiplication $b=P_{m}^{t} c$, where

$$
P_{m}^{t}=\left(\begin{array}{cc}
P_{\frac{m}{2}} & \frac{1}{2 m} I_{\frac{m}{2}} \\
\frac{-1}{2 m} I_{\frac{m}{2}} & O_{\frac{m}{2}}
\end{array}\right), \quad P_{2}^{t}=\left(\begin{array}{cc}
\frac{1}{2} & \frac{1}{4} \\
\frac{-1}{4} & 0
\end{array}\right)
$$

and $I_{m}$ and $O_{m}$ are the unit matrix and zero matrix of order $m$, respectively. (For details see $[6,2]$.$) Now, if f(x, y)$ has a Walsh series with coefficients $c_{i j}$ and its integral has a truncated Walsh series with coefficients of $b_{i j}$, such that

$$
\int_{0}^{x} \int_{0}^{y} f(t, s) d t d s=\int_{0}^{x} \int_{0}^{y} \sum_{i=0}^{m-1} \sum_{j=0}^{m-1} c_{i j} W_{i}(t) W_{j}(s) d t d s=\sum_{i=0}^{m-1} \sum_{j=0}^{m-1} b_{i j} W_{i}(x) W_{j}(y),
$$


we will show that the integration is performed by system of equations $b=\mathbf{P}_{m}^{\prime} c$. The solution process of this equation leads to a linear system in which the solution at the dyadic grid points is recovered by a method which requires as an input the initial value and average value of the function over the subintervals $\left[\frac{i-1}{m}, \frac{i}{m}\right]$ and $\left[\frac{j-1}{m}, \frac{j}{m}\right]$ and gives the approximate values of the solution at the grid points. In the next section, the two-dimensional operational matrix of integration is constructed.

\subsection{Operational matrix}

Before giving a more details of the Walsh approximation for two-dimensional integral equations, we obtain a general formula for integration of two-dimensional Walsh functions. The integration process of a step function is defined as: $\iint W_{0}(t) W_{0}(s) d t d s$. We will show that, the function $f$ can be expressed by a Walsh series. However, the coefficients $c_{i}$ of the Walsh series for the function $f(x)$ are given by $c_{m}=\frac{1}{m} W_{m} f_{m}$, and so for the function $f(x, y)$, the double Walsh coefficients are:

$$
c_{m}=\frac{1}{m^{2}} W_{m} f_{m} W_{m}
$$

We may set $m=2$, therefore:

$$
x y=c_{00} W_{0}(x) W_{0}(y)+c_{01} W_{0}(x) W_{1}(y)+c_{10} W_{1}(x) W_{0}(y)+c_{11} W_{1}(x) W_{1}(y),
$$

where the coefficients $c_{i j}$ are calculated as follows:

$$
\left(\begin{array}{ll}
c_{00} & c_{01} \\
c_{10} & c_{11}
\end{array}\right)=\frac{1}{4}\left(\begin{array}{cc}
1 & 1 \\
1 & -1
\end{array}\right)\left(\begin{array}{cc}
\frac{1}{16} & \frac{3}{16} \\
\frac{3}{16} & \frac{9}{16}
\end{array}\right)\left(\begin{array}{cc}
1 & 1 \\
1 & -1
\end{array}\right)=\left(\begin{array}{cc}
\frac{1}{4} & \frac{-1}{8} \\
\frac{-1}{8} & \frac{1}{16}
\end{array}\right) .
$$

Similarly, we can evaluate the Walsh series coefficients of the integration of functions $W_{0}(s) W_{1}(t), W_{1}(s) W_{0}(t)$ and $W_{1}(s) W_{1}(t)$. The operational matrix $\mathbf{P}_{2}^{\prime}$ with some changes in entries for the integration process $\int_{0}^{x} \int_{0}^{y} W_{i}(t) W_{j}(s) d t d s$, can be expressed in a block matrix form:

$$
\mathbf{P}_{4}^{\prime}=\left(\begin{array}{cc|cc}
\frac{1}{4} & \frac{1}{8} & \frac{1}{8} & \frac{1}{16} \\
\frac{-1}{8} & 0 & \frac{-1}{16} & 0 \\
\hline \frac{-1}{8} & \frac{-1}{16} & 0 & 0 \\
\frac{1}{16} & 0 & 0 & 0
\end{array}\right)=\left(\begin{array}{c|c|c}
\frac{1}{8} P_{2}^{t} & \frac{1}{4} P_{2}^{t} \\
\hline \frac{-1}{4} P_{2}^{t} & O_{2}
\end{array}\right)_{4 \times 4} .
$$

It is straightforward to show that the general form for the operational matrix $\mathbf{P}^{\prime}$ of order $m^{2}$ (which is positive integer power of 2) can be obtained as:

$$
\mathbf{P}_{m^{2}}^{\prime}=\left(\begin{array}{c|c|c|c}
\frac{1}{2} P_{m}^{t} & \frac{2}{m} \mathbf{P}_{\frac{m^{2}}{8}} & \frac{1}{m} \mathbf{P}_{\frac{m^{2}}{4}} & \frac{1}{2 m} \mathbf{P}_{\frac{m^{2}}{2}} \\
\hline \frac{-2}{m} \mathbf{P}_{\frac{m^{2}}{8}} & \mathbf{O}_{\frac{m^{2}}{8}} & \\
\hline \frac{-1}{m} \mathbf{P}_{\frac{m^{2}}{4}} & \mathbf{O}_{\frac{m^{2}}{4}} & \\
\hline \frac{-1}{2 m} \mathbf{P}_{\frac{m^{2}}{2}} & \mathbf{O}_{\frac{m^{2}}{2}}
\end{array}\right)_{m^{2} \times m^{2}}
$$


where $\mathbf{P}_{\frac{m^{2}}{2}}$ and $\mathbf{O}_{\frac{m^{2}}{2}}$ are the following matrices:

$$
\mathbf{P}_{\frac{m^{2}}{2}}=\left(\begin{array}{cccc}
P_{m}^{t} & O_{m} & \ldots & O_{m} \\
O_{m} & P_{m}^{t} & \ldots & O_{m} \\
\vdots & & \vdots \\
O_{m} & O_{m} & \ldots & P_{m}^{t}
\end{array}\right)_{\frac{m^{2}}{2} \times \frac{m^{2}}{2}} \quad, \quad \mathbf{O}_{\frac{m^{2}}{2}}=\left(\begin{array}{ccc}
O_{m} & \ldots & O_{m} \\
O_{m} & \ldots & O_{m} \\
\vdots & & \vdots \\
O_{m} & \ldots & O_{m}
\end{array}\right)_{\frac{m^{2}}{2} \times \frac{m^{2}}{2}}
$$

\section{The Numerical Analysis of the Scheme}

As a consequence of the previous section, here we derive formulas for numerical solvability of linear integral equation (1.1) based on double Walsh approximation and the two-dimensional operational matrix of integration. The first task is to replace all functions in (1.1) with their Walsh series as follows:

$$
u(x, y)=\sum_{i=0}^{\infty} \sum_{j=0}^{\infty} c_{i j} W_{i}(x) W_{j}(y), \quad c_{i j}=\int_{0}^{1} \int_{0}^{1} u(x, y) W_{i}(x) W_{j}(y) d x d y
$$

and

$$
f(x, y)=\sum_{i=0}^{\infty} \sum_{j=0}^{\infty} c_{i j}^{\prime} W_{i}(x) W_{j}(x), \quad c_{i j}^{\prime}=\int_{0}^{1} \int_{0}^{1} f(x, y) W_{i}(x) W_{j}(y) d x d y .
$$

The kernel $k(x, y, s, t)$ is approximated by a fourth order Walsh series:

$$
k(x, y, s, t)=\sum_{i=0}^{\infty} \sum_{j=0}^{\infty} \sum_{m=0}^{\infty} \sum_{n=0}^{\infty} k_{i j m n} W_{i}(x) W_{j}(x) W_{m}(s) W_{n}(t),
$$

where

$$
k_{i j m n}=\int_{0}^{1} \int_{0}^{1} \int_{0}^{1} \int_{0}^{1} k(x, y, s, t) W_{i}(x) W_{j}(y) W_{m}(s) W_{n}(t) d x d y d s d t .
$$

Following Fine [9], the $m=2^{n}$ th partial sum of the Walsh series of a function $f$ is a piecewise constant, equal to the $L^{1}$ mean of $f$ on each subinterval $\left[\frac{i-1}{m}, \frac{i}{m}\right]$. In this case the coefficients $c_{i j}^{\prime}$ and $k_{i j m n}$ of the Walsh series are:

$$
c_{i j}^{\prime}=\sum_{i=0}^{m-1} \sum_{j=0}^{m-1} \frac{1}{m^{2}} W_{i j} \tilde{f}_{i j} W_{i j}
$$

where $\tilde{f}_{i j}$ is the average value of the function $f(x, y)$ in all subintervals and $W_{i j}$ is the value of the $i$ th Walsh function in the $j$ th subinterval. Also, we get:

$$
K_{m^{2}}=\frac{1}{m^{4}} W_{m^{2}} \widetilde{K}_{m^{2}} W_{m^{2}}
$$

Dividing the interval $[0,1)$ into $m$ subintervals along the $x$-axis and the $y$-axis, respectively, we replace all functions by their Walsh series and integrate them in 
appropriate moments over the grids of the form $\left[\frac{i-1}{m}, \frac{i}{m}\right)$ and $\left[\frac{j-1}{m}, \frac{j}{m}\right)$. Finally, the following matrix equation is obtained:

$$
\begin{aligned}
W_{m} C_{m} W_{m}^{\prime}= & W_{m} C_{m}^{\prime} W_{m}^{\prime}+\sum_{l=0}^{m-1} \sum_{k=0}^{m-1} \sum_{g=0}^{m-1} \sum_{h=0}^{m-1} \sum_{p=0}^{m-1} \sum_{q=0}^{m-1} k_{g h p q} c_{l k} \\
& \times\left[\begin{array}{ccc}
W_{i+1,1}^{\prime} a_{11} W_{j+1,1} & \cdots & W_{i+1,1}^{\prime} a_{1 m} W_{j+1, m} \\
W_{i+1,2}^{\prime} a_{21} W_{j+1,1} & \cdots & W_{i+1,2}^{\prime} a_{2 m} W_{j+1, m} \\
\vdots & \vdots & \vdots \\
W_{i+1, m}^{\prime} a_{m 1} W_{j+1,1} & \cdots & W_{i+1, m}^{\prime} a_{m m} W_{j+1, m}
\end{array}\right] .
\end{aligned}
$$

Note that in our innovate matrix, the $W_{i+1, t}^{\prime}(1 \leq t<m)$ is the $(i+1, t)$ th entry of the Walsh matrix along the $x$-axis, $W_{i+.1, t}$ is the $(i+1, t)$ th entry of the Walsh matrix along the $y$-axis and $a_{i j}(1 \leq i, j<m)$ is defined as follows:

$$
W_{m} \mathbf{P}_{1} W_{m}^{\prime}=\left[\begin{array}{ccc}
a_{11} & \cdots & a_{1 m} \\
\vdots & \vdots & \vdots \\
a_{m 1} & \cdots & a_{m m}
\end{array}\right]
$$

For computing the above matrix, we need to calculate:

$$
\int_{0}^{x} \int_{0}^{y} W_{l}(s) W_{k}(t) W_{p}(s) W_{q}(t) d s d t .
$$

Following [11, p. 1159], using the multiplication rule of two-dimensional Walsh series, we take $z=l \otimes p$ and $v=k \otimes q$, then we must compute the double integral $\int_{0}^{x} \int_{0}^{y} W_{z}(s) W_{v}(t) d s d t$. Note that one of the columns of operational matrix $\mathbf{P}^{\prime}$ for $l, k, p, q=0, \ldots, m-1$ is each of integration process. Changing each $m^{2}$ entries of column of operational matrix to the $m \times m$ matrix, the matrix $\mathbf{P}_{1}$ is obtained.

Finally, we obtain a linear system of $m^{2}$ equation with $m^{2}$ unknown coefficients, which gives the Walsh coefficients. The reliability and efficiency of the proposed scheme are demonstrated by some numerical experiments, in the next section.

\section{Numerical Experiments}

Test problem 1. Consider the following two-dimensional Volterra integral equation:

$$
u(x, y)=x^{2}+y^{2}-\frac{y^{3}}{3} x-\frac{x^{3}}{3} y+\int_{0}^{x} \int_{0}^{y} u(t, s) d t d s, \quad(x, y) \in[0,1] \times[0,1],
$$

with the exact solution $u(x, y)=x^{2}+y^{2}$. Let $u_{N}(x, y)$ be the approximate solution of the equation which is approximated by the truncated double Walsh series:

$$
u_{N}(x, y)=\sum_{i=0}^{m-1} \sum_{j=0}^{m-1} c_{i j} W_{i}(x) W_{j}(y)
$$




\begin{tabular}{c|c|c}
\hline$m$ & $h$ & Max error \\
\hline 2 & $\frac{1}{2}$ & $2.00 \times 10^{-3}$ \\
4 & $\frac{1}{4}$ & $1.10 \times 10^{-5}$ \\
8 & $\frac{1}{8}$ & $6.80 \times 10^{-9}$ \\
\hline
\end{tabular}

We choose a uniform partition of the unit interval $[0,1)$ and set $h=\frac{1}{m}$ for $m=$ $2,4,8, \ldots$ The $m^{2}$ Walsh coefficients of approximate solution have been obtained using the linear system of equation (3.1). All computations were carried out in double precision arithmetic with Maple ${ }^{\circledR}$ software. The maximum errors of the scheme are given in Table 1.

However, better approximation is expected by choosing a larger values of $m$.

Test problem 2. (From [16]) Consider the first kind of Volterra integral equation:

$f(x, y)=\int_{0}^{x} \int_{0}^{y}(\sin (x+t)+\sin (y+s)+3) u(t, s) d t d s, \quad(x, y) \in[0,1] \times[0,1]$,

and choose $f(x, y)$ such that the exact solution is $u(x, y)=\cos (x+y)$.

The $L_{\infty}$ errors in some subintervals for $m=4$ are of special interest and can be compared with those obtained by previous work [16]. The matrix form of above equation for $m=4$ is as follows:

$$
\begin{aligned}
& W_{4} C_{4} W_{4}^{\prime}=W_{4} C_{4}^{\prime} W_{4}^{\prime}+\sum_{l=0}^{3} \sum_{k=0}^{3} \sum_{i=0}^{3} \sum_{j=0}^{3} \sum_{p=0}^{3} \sum_{q=0}^{3} k_{i j p q} c_{l k} \\
& \times\left[\begin{array}{llll}
W_{i+1,1}^{\prime} a_{11} W_{j+1,1} & W_{i+1,1}^{\prime} a_{12} W_{j+1,2} & W_{i+1,1}^{\prime} a_{13} W_{j+1,3} & W_{i+1,1}^{\prime} a_{14} W_{j+1,4} \\
W_{i+1,2}^{\prime} a_{21} W_{j+1,1} & W_{i+1,2}^{\prime} a_{22} W_{j+1,2} & W_{i+1,2}^{\prime} a_{23} W_{j+1,3} & W_{i+1,2}^{\prime} a_{24} W_{j+1,4} \\
W_{i+1,3}^{\prime} a_{31} W_{j+1,1} & W_{i+1,3}^{\prime} a_{32} W_{j+1,2} & W_{i+1,3}^{\prime} a_{33} W_{j+1,3} & W_{i+1,3}^{\prime} a_{34} W_{j+1,4} \\
W_{i+1,4}^{\prime} a_{41} W_{j+1,1} & W_{i+1,4}^{\prime} a_{42} W_{j+1,2} & W_{i+1,4}^{\prime} a_{43} W_{j+1,3} & W_{i+1,4}^{\prime} a_{44} W_{j+1,4}
\end{array}\right] .
\end{aligned}
$$

We take $h=0.25$, and $m_{i}=\left[\frac{i-1}{m}, \frac{i}{m}\right], n_{j}=\left[\frac{j-1}{m}, \frac{j}{m}\right]$, so, the results for different values of $i=j=1,2,3,4$ are obtained.

\begin{tabular}{cc}
\hline$i(=j)$ & $h=0.25$ \\
\hline 1 & $9.21 \times 10^{-4}$ \\
2 & $7.19 \times 10^{-4}$ \\
3 & $3.02 \times 10^{-3}$ \\
4 & $1.71 \times 10^{-5}$ \\
\hline$\|e(h)\|_{\infty}$ & $3.02 \times 10^{-3}$ \\
\hline
\end{tabular}


For the Walsh approximation scheme, the errors on the subintervals are mainly less than the corresponding errors at the other grid points for the Euler and trapezoidal methods. (For details see [16].)

\section{Conclusion}

The intent of this note was to confirm the Walsh approximation for numerical solution of general two-dimensional Volterra integral equations. The reliability and efficiency of the scheme are demonstrated by some numerical experiments. Due to the nature of Walsh functions, the process of solution mainly depends on $m$, where for the larger values of $m$, we have some restrictions dealing with large matrices. However, following [19], using the generalization of the single-term Walsh series strategy we can address this problem in the proposed scheme that will be addressed in an upcoming paper.

\section{References}

1. B. A. Beltyukov and L. N. Kuznechikhina, A Runge-Kutta method for the solution of two-dimensional nonlinear Volterra integral equations, Diff. Eq. 12 (1976) 1169-1173.

2. W. F. Blyth, R. L. May and P. Widyaningsih, Volterra integral equations solved in Fredholm form using Walsh functions, Anziam J. 45(E) (2004) 269-282.

3. W. F. Blyth and V. Uljanov, Sloan iteration and Richardson extrapolation for Walsh series solutions of integral equations, Anziam J. 46(E) (2005) 690-703.

4. H. Brunner and J. P. Kauthen, The numerical solution of two-dimensional Volterra integral equations by collocation and iterated collocation, IMA J. Numer. Anal. 9 (1989) 47-59.

5. V. Carutasu, Numerical solution of two dimensional nonlinear Fredholm integral equations of the second kind by spline functions, General Math. 9 (2001) 31-48.

6. C. F. Chen and C. H. Hsiao, A Walsh series direct method for solving variational problems, J. Franklin Inst. 300 (1975) 265-280.

7. E. K. Chui, An Introduction to Wavelets, Academic Press, 1992.

8. H. G. Dobner, Bounds for the solution of hyperbolic problems, Computing 38 (1987) 209-218.

9. N. J. Fine, On the Walsh functions, Trans. Amer. Math. Soc. 65 (1949) 372-414.

10. J. E. Gibbs and H. A. Gebbie, Application of Walsh function to transform spectroscopy, Nature 1(224) (1969) 1012-1013.

11. H. Guoqiang and Z. Liqing, Asymptotic error expansion for the trapezoidal Nystrom method of linear Volterra-Fredholm equations, J. Comput. Appl. Math. 51(3) (1994) 339-348.

12. H. Guoqiang, K. Hayami, K. Sugihara and W. Jiong, Extrapolation method of iterated collocation solution for two-dimensional nonlinear Volterra integral equations, Appl. Math. Comput. 112 (2001) 49-61.

13. H. Guoqiang and R. Wang, The extrapolation method for two-dimensional Volterra integral equations based on the asymptotic expansion of iterated Galerkin solutions, J. Integral Eq. Appl. 13(1) (2001) 15-34.

14. H. Guoqiang an and L. Q. Zhang, Asymptotic error expansion of two-dimensional Volterra integral equations by iterated collocation, Appl. Math. Comput. 61 (1994) 269-285. 
15. C. H. Hsiao and C. F. Chen, Solving integral equations via Walsh functions, Comput. Elect. Eng. 6 (1979) 279-292.

16. S. McKee, T. Tang and T. Diogo, An Euler-type method for two dimensional Volterra integral equations of the first kind, IMA J. Numer. Anal. 20 (2000) 423-440.

17. F. Picher, Walsh function and optimal linear systems, Proc. Walsh function Symp., Nav. Res. Labs., Washington D.C., (1970) 17-22.

18. M. Razzaghi and J. Nazarzadeh, Walsh functions, Willey Encyclopedia Electr. Eng. 23 (1999) 429-440.

19. B. Sepehrian and M. Razzaghi, Single-term Walsh series method for the Volterra integro-differntial equations, Eng. Anal. Bound. Elements 28 (2004) 1315-1319.

20. B. G. Sloss and W. F. Blyth, A Variational method using Walsh functions, Nonlinear Anal. 32 (1998) 549-561.

21. B. G. Sloss and W. F. Blyth, The computational efficiency of a spectral Walsh function method, J. Franklin Inst. 335 (1998) 1157-1170.

22. B. G. Sloss and W. F. Blyth, A Walsh function method for a nonolinear Volterra integral equation, J. Franklin Inst. 340 (2003) 25-41.

23. P. Singh, A note on the solution of two-dimensional Volterra integral equations by splines, Indian J. Math. 18 (1976) 61-64.

24. F. Weisz, Cesaro summability of two-dimensional Walsh Fourier series, Trans. Amer. Math. Soc. 348 (1996) 2169-2181. 